\title{
Ionization degree of the electron-hole plasma in semiconductor quantum wells
}

\author{
M. E. Portnoi* and I. Galbraith \\ Physics Department, Heriot-Watt University, Edinburgh EH14 4AS, United Kingdom
}

(Received 7 January 1999)

\begin{abstract}
The degree of ionization of a nondegenerate two-dimensional electron-hole plasma is calculated using the modified law of mass action, which takes into account all bound and unbound states in a screened Coulomb potential. Application of the variable phase method to this potential allows us to treat scattering and bound states on the same footing. Inclusion of the scattering states leads to a strong deviation from the standard law of mass action. A qualitative difference between midgap and wide-gap semiconductors is demonstrated. For wide-gap semiconductors at room temperature, when the bare exciton binding energy is of the order of $k_{B} T$, the equilibrium consists of an almost equal mixture of correlated electron-hole pairs and uncorrelated free carriers. [S0163-1829(99)03532-8]
\end{abstract}

\section{INTRODUCTION}

The drive for ever higher storage capacity has led to the development of semiconductor lasers operating in the blue spectral region, based on $\mathrm{ZnSe}$ (Ref. 1) and GaN. ${ }^{2}$ Along with the large energy gap of these materials comes a large exciton binding energy, of the same order as $k_{B} T$ at room temperature. As has been well known since the $1970 \mathrm{~s},{ }^{3}$ excitonic gain processes are important in wide-gap semiconductors, and their importance is further enhanced in quantum-well structures where the binding energy may be considerably larger than $k_{B} T$ (e.g., $\sim 35 \mathrm{meV}$ in $\mathrm{Zn}_{x} \mathrm{Cd}_{1-x} \mathrm{Se} / \mathrm{ZnSe}$ quantum wells).

Theoretical treatments of GaAs- and InP-based lasers are well established using a microscopic many-body approach based on linear-response theory. ${ }^{4}$ Screening and band-gap renormalization effects are included, assuming that the injected carriers form a completely ionized electron-hole plasma. Such treatments have been successful in explaining many of the observed features of mid-infrared laser diodes. Complex valence-band effects and strain effects as well as carrier thermalization effects have all been included at various levels of complexity. In this way, a relatively complete understanding exists for the basic operation of these lasers.

In wide-gap semiconductors, however, the strong Coulomb interaction leads to the existence of bound-exciton states, which persist even at elevated densities and temperatures. As such, the conventional assumption that the inversion is in the form of an electron-hole plasma with no excitons present deserves closer examination. A self-consistent description where both bound and unbound states are treated on an equal footing is required. Unfortunately, as far as we are aware no comprehensive theoretical treatment of this problem exists. Treatments based on bosonic exciton operators have been proposed, ${ }^{5}$ but this approach breaks down at high injection when the screening of the Coulomb potential weakens the binding and produces a population of unbound scattering states, which clearly do not exhibit bosonic character. On the other hand, a treatment based around fermionic electron and hole operators is complex when higher-order excitonic correlations are important. ${ }^{6,7}$ A natural concept in considering this issue is the degree of ionization in the inter- acting electron-hole plasma, and in this paper we calculate this for a two-dimensional (2D) plasma. We focus on two dimensious for two reasons. First, most modern semiconductor lasers are fabricated in quantum-well heterostructures. Second, the presence of at least one bound state in the attractive $2 \mathrm{D}$ potential requires a nonperturbative treatment of the screened Coulomb interaction.

We will be mostly interested in the plasma properties induced by the pair Coulomb interaction between charged particles, neglecting band-gap renormalization and phase-space filling effects, which have been extensively studied in both three-dimensional (3D) and 2D cases. ${ }^{8,9}$ These effects can be neglected only in the low-density (nondegenerate) limit, which is defined in $2 \mathrm{D}$ by the inequality

$$
n \lambda_{M}^{2} / g<1
$$

where $n$ is the 2D carrier density, $g$ is the spin degeneracy factor of $2 \mathrm{D}$ particles, and $\lambda_{M}=\left(2 \pi \hbar^{2} / M k_{B} T\right)^{1 / 2}$ is the thermal wavelength. For the two-component plasma the lighter carrier (usually electron) effective mass must be used to evaluate the thermal wavelength to ensure that condition (1) is valid for both types of carriers. Inequality (1) provides that the motion of excitons can also be considered as classical. For GaAs at room temperature $\lambda_{M_{e}} \approx 1.66 \times 10^{-6} \mathrm{~cm}$, electron-spin degeneracy $g_{e}=2$, and condition (1) is satisfied for $n \leqq 7.2 \times 10^{11} \mathrm{~cm}^{-2}$. The electron effective mass in wide-gap semiconductors is usually at least two times larger than in midgap semiconductors; therefore, condition (1) is valid over a wider range of carrier densities [e.g., for $\mathrm{ZnSe}$ at room temperature, inequality (1) is satisfied for $n \lesssim 1.7$ $\left.\times 10^{12} \mathrm{~cm}^{-2}\right]$. Thus the nondegenerate (Boltzmann) limit is not only a convenient approximation, in which the Coulomb interaction is not hidden by the band-filling effects, but it also gives a realistic picture of the electron-hole plasma in wide-gap semiconductors at room temperature and moderate carrier densities. Lasing at anomalously low densities (below the Mott density) has been reported in $\mathrm{Zn}_{x} \mathrm{Cd}_{1-x} \mathrm{Se} / \mathrm{ZnSe}$ quantum wells. ${ }^{10}$

Following an approach applied in three dimensions to nuclear matter, ${ }^{11}$ an ionic plasma, ${ }^{12}$ and the electron-hole 
system in excited semiconductors, ${ }^{13}$ we divide the total electron (hole) density between two terms:

$$
n_{a}=n_{a}^{0}+n_{a}^{\text {corr }} .
$$

The first term $n_{a}^{0}$ is the density of uncorrelated quasiparticles with renormalized energies. This term is that part of the total density which is independent of the interparticle interaction (see Appendix B). All correlation effects both in the bound and continuum states are incorporated into the second term $n_{a}^{\text {corr }}$, which is called the correlated density. The lower index in Eq. (2) is a species index, $a=e$ for electrons and $a=h$ for holes. It is also useful to introduce the degree of ionization of the electron-hole plasma,

$$
\alpha=\frac{n_{e}^{0}}{n_{e}}=\frac{n_{e}^{0}}{n_{e}^{0}+n_{e}^{\text {corr }}},
$$

which characterizes the deviation of the thermodynamic properties of the electron-hole plasma from those of the ideal gas $(\alpha=1)$. The knowledge of the degree of ionization is essential in determining the dominant lasing mechanism. When $\alpha$ is close to unity, the main lasing mechanism is stimulated emission from the free-carrier plasma, for lower values of $\alpha$ several excitonic gain processes have to be considered. ${ }^{14}$

In the nondegenerate limit there is no need to go beyond two-particle correlations. This allows us to separate clearly the role of the inter-particle Coulomb interaction from the phase-space filling effects. In this limit, the correlated and uncorrelated densities are related by

$$
n_{a}^{\text {corr }}=\sum_{b} n_{a}^{0} n_{b}^{0} \frac{2 \pi \beta \hbar^{2}}{\mu_{a b}} Z_{a b},
$$

where $\beta=1 /\left(k_{B} T\right), \mu_{a b}=M_{a} M_{b} /\left(M_{a}+M_{b}\right)$ is the reduced effective mass, and $Z_{a b}$ is the two-body interaction part of the partition function. This relationship is derived in Appendix B. Note that due to charge neutrality the total electronhole density $n_{e}=n_{h}=n$ is independent of species, whereas $n_{e}^{0} \neq n_{h}^{0} \quad$ and $n_{e}^{\text {corr }} \neq n_{h}^{\text {corr }}$ if electrons and holes have different effective masses.

The electron-hole part of the partition function which exhibits bound states (excitons) is given by

$$
\begin{aligned}
Z_{e h}= & \sum_{m, \nu} \exp \left(-\beta E_{m, \nu}\right)+\frac{1}{\pi} \int_{0}^{\infty}\left(\sum_{m=-\infty}^{\infty} \frac{d \delta_{m}(k)}{d k}\right) \\
& \times \exp \left(-\beta \frac{\hbar^{2} k^{2}}{2 \mu_{e h}}\right) d k,
\end{aligned}
$$

where $m \hbar$ is the projection of the angular momentum onto the axis normal to the plane of $2 \mathrm{D}$ motion $(m=0, \pm 1$, $\pm 2, \ldots), \hbar^{2} k^{2} /\left(2 \mu_{e h}\right)$ is the energy of the relative motion of the unbound (scattered) electron and hole, $\hbar k$ is the magnitude of the relative motion momentum, $\delta_{m}(k)$ are the 2D scattering phase shifts, ${ }^{17,18} E_{m, \nu}$ are the bound-state energies (index $\nu$ enumerates bound states with given $m$ ), and the double sum in the first term ranges only over bound states. Equation (5) is the 2D analog of the Beth-Uhlenbeck formula ${ }^{19}$ and it can be derived in the same fashion as in the 3D case, as shown in Appendix B.
The scattering (integral) term on the right-hand side of Eq. (5) gives the contribution to $Z_{e h}$ of the continuum part of the energy spectrum. This term is usually neglected in calculations of the ionization degree of the electron-hole plasma. ${ }^{3,14-16}$ In what follows we will show that at high enough temperature the scattering term is comparable to the bound-state sum and indeed this term has to be taken into account to ensure continuity of the partition function whenever bound states disappear with increasing screening. ${ }^{20}$

The electron-electron and hole-hole parts of the partition function $Z_{e e}$ and $Z_{h h}$ contain the scattering term only. To calculate $Z_{a a}$ one must take into account the Pauli exclusion principle for identical particles, which modifies the sum over $m$. The electron-electron (hole-hole) part of the partition function is given by (see Appendix B)

$$
Z_{a a}=\frac{1}{2 \pi} \sum_{m=-\infty}^{\infty}\left\{2-(-1)^{m}\right\} \int_{0}^{\infty} \frac{d \delta_{m}(k)}{d k} \exp \left(-\beta \frac{\hbar^{2} k^{2}}{M_{a}}\right) d k .
$$

Here we assume that both electron and hole states in quantum wells are two-fold degenerate. The only difference between $Z_{h h}$ and $Z_{e e}$ arises from the difference between electron and hole effective masses.

Equations (2)-(6) provide a connection between the total electron-hole density $n$ and uncorrelated quasiparticle densities $n_{e}^{0}$ and $n_{h}^{0}$. The quasiparticle densities in turn govern the screening ${ }^{13}$ and therefore the strength of interaction between particles, which uniquely defines the set of binding energies and scattering phase shifts which enter Eqs. (5) and (6) for the two-body partition functions. These partition functions in turn define the ratio between $n_{a}^{0}$ and $n_{a}^{\text {corr }}$ via Eq. (4). Thus, to find the degree of ionization of the electron-hole plasma, one must solve the system of equations (2)-(6) selfconsistently, together with a reasonable model of the screened interaction.

In Sec. II we discuss the statically screened Coulomb potential which we use to model the interaction between particles in an exciton/electron-hole plasma, and present results from the application of the variable phase method ${ }^{22}$ to scattering and bound states in this potential. In Sec. III we present and discuss the results of calculations of partition functions and the degree of ionization of the electron-hole plasma. In Appendix A we derive the basic equations of the variable phase method, which is used for calculation of scattering phase shifts and binding energies. The 2D analog of the Beth-Uhlenbeck formula and the modified law of mass action are derived in Appendix B.

\section{STATICALLY SCREENED COULOMB POTENTIAL}

There is an extensive literature dealing with different aspects of the screened Coulomb interaction in 2D systems. ${ }^{23}$ In this paper we model this interaction by the well-known Thomas-Fermi expression for a statically screened Coulomb potential, ${ }^{17}$

$$
\begin{aligned}
V_{s}(\rho) & =\mp \frac{e^{2}}{\epsilon} \int_{0}^{\infty} \frac{q J_{0}(q \rho)}{q+q_{s}} d q \\
& =\mp \frac{e^{2}}{\epsilon}\left\{\frac{1}{\rho}-\frac{\pi}{2} q_{s}\left[\mathbf{H}_{0}\left(q_{s} \rho\right)-N_{0}\left(q_{s} \rho\right)\right]\right\},
\end{aligned}
$$




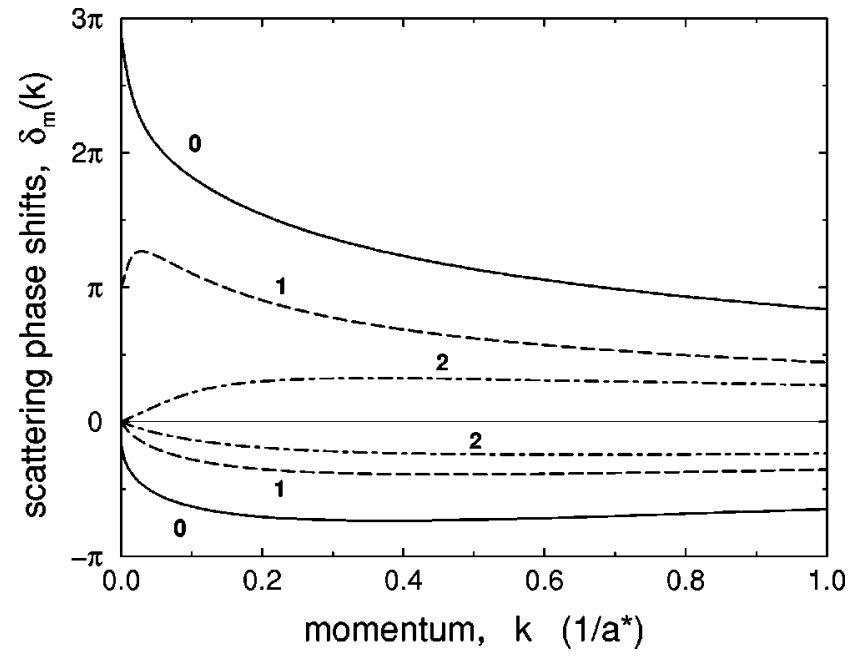

FIG. 1. Scattering phase shifts vs the in-plane wave vector $k$ (in units of inverse Bohr radius $1 / a^{*}$ ) for a 2D particle in a screened Coulomb potential [Eq. (7)]. The screening wave number $q_{s}$ $=0.2 / a^{*}$. For the attractive potential all phase shifts are positive, and for the repulsive potential they are negative. Numbers show $m$ values.

where $q_{s}$ is the 2D screening wave number (which depends on temperature and carrier density), $\epsilon$ is the static dielectric constant of the semiconductor, and $J_{0}(x), N_{0}(x)$, and $\mathbf{H}_{0}(x)$ are the Bessel, Neumann, and Struve functions, respectively. The upper sign in Eq. (7) is for electron-hole attraction, and the lower sign is for electron-electron or holehole repulsion.

Being the long-wavelength, static limit of the randomphase approximation for a purely $2 \mathrm{D}$ case, ${ }^{24,25} \mathrm{Eq}$. (7) is the simplest model for the screened Coulomb potential in two dimensions. Nevertheless, this expression reflects the fact that the statically screened potential in two dimensions decreases at large distances slower than in the 3D case (as a power law rather than exponentially). Despite numerous realistic corrections, ${ }^{23,26,27}$ Eq. (7) remains the most widely used approximation for the 2D screening, especially for the screened exciton problem. ${ }^{16,28-31}$ Optically active $(m=0)$ bound states in the attractive, statically screened Coulomb potential [upper sign in Eq. (7)] have been studied using a variational method, ${ }^{28,29}$ by a numerical procedure based on a shooting method, ${ }^{30}$ and more recently using the WKB approximation $^{31}$ and perturbation theory. ${ }^{16}$ As mentioned above, for the partition function calculation, all states are needed, bound and unbound, optically active and inactive. None of the above methods is suitable for analysis of shallow bound states and low-energy scattering states.

We use for calculation of the scattering phase shifts and bound state energies entering Eqs. (5) and (6) the 2D formulation ${ }^{32}$ of the variable phase method. ${ }^{22}$ In this method the scattering phase shift and the function defining boundstate energies can be obtained as the large distance limit of the phase function, which satisfies the first-order, nonlinear Riccati equation originating from the radial Schrödinger equation (see Appendix A). The variable phase method is especially effective for calculation of the shallow-state binding energies and low-energy scattering phase shifts.

In Fig. 1 we show the $k$ dependence of the scattering phase shifts for the attractive and repulsive Thomas-Fermi potentials [both signs of Eq. (7)] with the screening wavenumber $q_{s}=0.2 / a^{*}$, where $a^{*}=\epsilon \hbar^{2} /\left(\mu e^{2}\right)$ is the 3D exciton Bohr radius. The scattering phase shifts are negative for the repulsive potential and positive for the attractive potential. For the repulsive potential all zero-energy phase shifts vanish, $\delta_{m}(k=0)=0$ for all angular momenta $m$. For the attractive potential,

$$
\lim _{k \rightarrow 0} \delta_{m}(k)=\nu_{m} \pi
$$

where $\nu_{m}$ is the number of bound states. ${ }^{35}$ Equation (8) is the 2D analog of Levinson's theorem ${ }^{33}$ (see also Ref. 34), which connects the zero-energy scattering phase shift with the number of bound states for nonrelativistic particles in three dimensions. This theorem has been known for almost five decades; however, its applicability to the 2D scattering problem has been considered only recently. ${ }^{32,36-38}$

We recently ${ }^{32}$ used Levinson's theorem in the form of Eq. (8) for bound-state counting in the attractive Thomas-Fermi potential, Eq. (7), and found a remarkably simple relation between the number of bound states and the screening wave number $q_{s}$. With decreasing screening, bound states appear at critical values of the screening length given by the simple formula $^{32}$

$$
\left(\frac{1}{q_{s} a^{*}}\right)_{c}=\frac{(2|m|+\nu-1)(2|m|+\nu)}{2}, \nu=1,2, \ldots
$$

Equation (9) can be easily inverted, and the number of bound states for given $m$ and $q_{s}$ can be expressed as

$$
\nu_{m}=\max \left\{0, \nu_{0}-2|m|\right\},
$$

where

$$
\nu_{0}=\left[\frac{\sqrt{8 /\left(q_{s} a^{*}\right)+1}+1}{2}\right]
$$

is the number of bound states with $m=0$. Here, and in Eqs. (13) and (14), the bold square brackets designate the integer part of a number. For small $q_{s}$, Eq. (11) gives a number 2.5 times smaller than the WKB estimate ${ }^{31}$ for the maximum number of bound $s$ states. The Bargmann bound condition ${ }^{39}$ (restated for the 2D case ${ }^{17}$ ) for the attractive potential (7) is $\nu_{m}<1 /\left(m q_{s} a^{*}\right)$. This was also found to give a gross overestimate of the number of bound states.

The total number of bound states, $N_{b}$, for a given $q_{s} a^{*}$,

$$
N_{b}=\nu_{0}+2 \sum_{m=1}^{|m|_{\max }} \nu_{m},
$$

can also be found explicitly as follows. From Eq. (9) the maximum possible value of $|m|$ for the state which remains bound is

$$
|m|_{\max }=\left[\frac{\sqrt{8 /\left(q_{s} a^{*}\right)+1}-1}{4}\right]=\left[\frac{\nu_{0}-1}{2}\right] .
$$

Then the sum in Eq. (12) can be easily evaluated using Eqs. (10) and (13): 


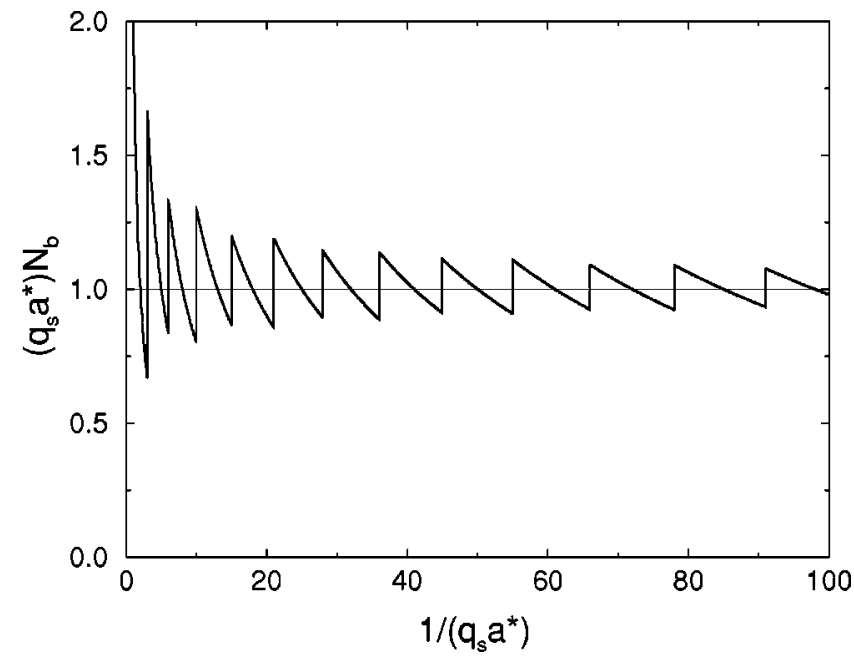

FIG. 2. The normalized number of bound states $\left(q_{s} a^{*}\right) N_{b}$ as a function of the inverse screening parameter, $1 /\left(q_{s} a^{*}\right)$, for $q_{s} a^{*}$ $\leqslant 1$.

$$
\begin{aligned}
N_{b} & =\nu_{0}+2|m|_{\max }\left(\nu_{0}-|m|_{\max }-1\right) \\
& =\nu_{0}+2\left[\frac{\nu_{0}-1}{2}\right]\left\{\nu_{0}-1-\left[\frac{\nu_{0}-1}{2}\right]\right\} .
\end{aligned}
$$

For small $q_{s} a^{*}$ (weakly screened potential) a simple approximate expression for the total number of bound states follows from substitution of Eq. (11) into Eq. (14):

$$
N_{b} \approx \frac{\nu_{0}^{2}}{2} \approx \frac{1}{q_{s} a^{*}}
$$

Thus, for the weakly screened Thomas-Fermi potential, the bound-state sum in the partition function [Eq. (5)] has a finite number of terms which is approximately equal to the screening radius $1 / q_{s}$ measured in units of the Bohr radius. The WKB estimate of the number of bound states ${ }^{31}$ gives a different (square root) dependence of $N_{b}$ on $1 /\left(q_{s} a^{*}\right)$ for small $q_{s} a^{*}$. The reason for this difference is that in Ref. 31 only $m=0$ states are considered, whereas all values of $m$ are needed to obtain the result of Eq. (15).

As the screening is reduced, $N_{b}$, given by Eq. (14), exhibits steps of ever increasing height. In order for the limit of Eq. (15) to be meaningful the step height should be smaller than $N_{b}$ itself, i.e., the normalized number of bound states, $N_{b} / N_{b}^{q_{s} \rightarrow 0}=\left(q_{s} a^{*}\right) N_{b}$, should converge to unity as $q_{s} a^{*}$ $\rightarrow 0$. As can be seen in Fig. 2, this number oscillates around unity with the amplitude of oscillations decreasing with increasing $1 /\left(q_{s} a^{*}\right)$. It can be shown that for $q_{s} a^{*} \rightarrow 0$ the amplitude of these oscillations is proportional to $\left(q_{s} a^{*}\right)^{1 / 2}$, and their period is proportional to $\left(q_{s} a^{*}\right)^{-1 / 2}$.

In order to calculate the partition function, the bound-state energies are required. These can also be obtained using the variable phase method, and the necessary equations are presented in Appendix A 2. Numerical results for the attractive screened Coulomb potential [upper sign in Eq. (7)] are presented in Fig. 3. In this figure the energies $E_{m, \nu}$ of the several lowest bound states of the screened exciton are shown as a function of the screening wave number $q_{s} a^{*}$. Here the energies are measured in effective exciton Rydberg [Ry*

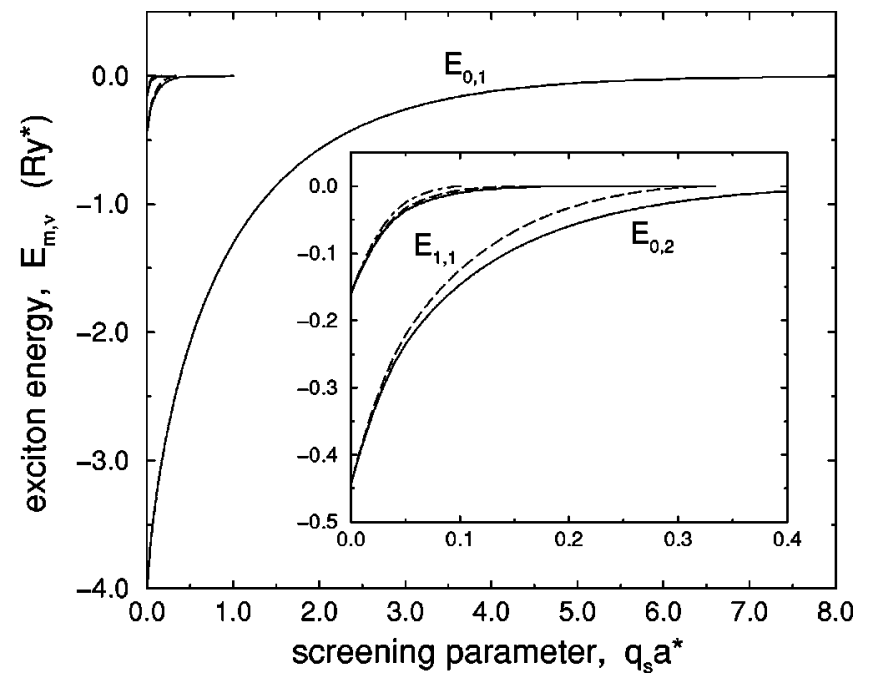

FIG. 3. The bound-state energies $E_{m, \nu}$ of the 2D exciton in exciton Rydberg units are shown as a function of the screening parameter $q_{s} a^{*}$ for different $m$ values. Solid lines show $m=0$ states $\left(E_{0,1}, E_{0,2}\right.$, and $\left.E_{0,3}\right)$; dashed lines show $m=1$ states $\left(E_{1,1}\right.$ and $\left.E_{1,2}\right)$; the dot-dashed line shows the lowest state with $m$ $=2\left(E_{2,1}\right)$. The inset is an enlargement near $q_{s} a^{*}=0$.

$\left.=\hbar^{2} /\left(2 \mu_{e h} a^{* 2}\right)\right]$, and we use the same classification of energy levels as in Ref. 32, i.e., each energy level is characterized by the angular momentum quantum number $m$ and another number $\nu$ which numerates different bound states for a given $m$, with $(\nu-1)$ being the number of nonzero nodes of the radial wave function. For $m=0$ ( $s$ states) the calculated energies are consistent with those obtained by J. Lee et al. ${ }^{29}$ using a variational method.

\section{PARTITION FUNCTIONS AND IONIZATION DEGREE}

Before we present the results of calculations of the partition functions and the ionization degree, we would like to discuss an important consequence of Levinson's theorem for the statistical mechanics of the 2D gas with an attractive interaction between its particles. The bound-state sum $Z_{\text {bound }}=\Sigma_{m, \nu} \exp \left(-\beta E_{m, \nu}\right)$, entering the two-body partition function in Eq. (5), exhibits jumps whenever bound states disappear with increasing screening. We will now show that these jumps do not give rise to unphysical discontinuities in the partition function if the scattering states are properly taken into account.

Integrating by parts the scattering term and using Levinson's theorem in the form of Eq. (8), we can rewrite Eq. (5) as

$$
\begin{aligned}
Z_{e h}= & \sum_{m, \nu}\left\{\exp \left(-\beta E_{m, \nu}\right)-1\right\}+\frac{2}{\pi q_{T}^{2}} \int_{0}^{\infty}\left(\sum_{m=-\infty}^{\infty} \delta_{m}(k)\right) \\
& \times \exp \left(-k^{2} / q_{T}^{2}\right) k d k
\end{aligned}
$$

where $q_{T}^{2}=2 \mu_{e h} k_{B} T / \hbar^{2}$. The modified bound-state sum [the first term in Eq. (16)] does not exhibit jumps whenever bound states disappear with increasing screening. For nonzero temperature the scattering integral [the second term in Eq. (16)] is also a smooth function of the interaction strength, which can be understood from Fig. 4. In this figure 


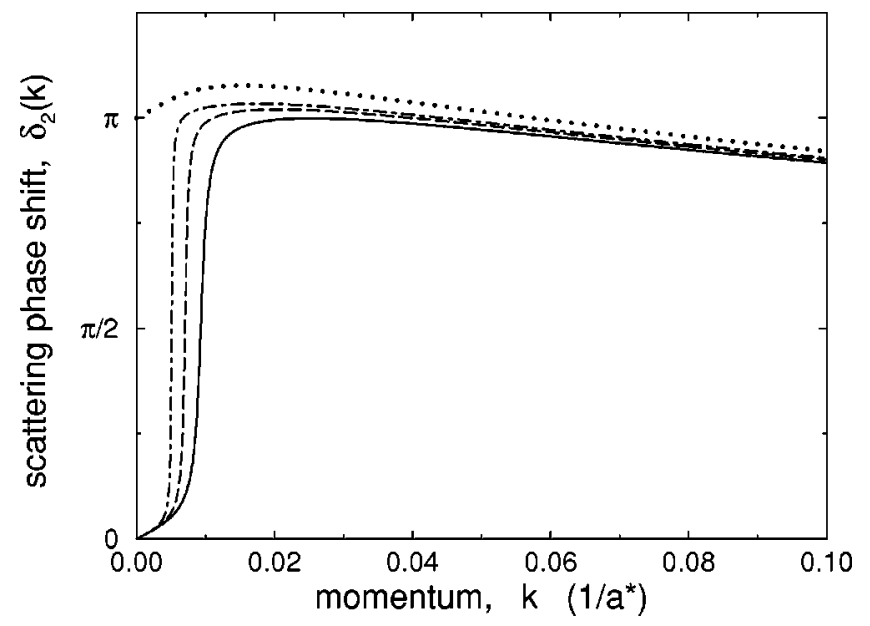

FIG. 4. The scattering phase shift $\delta_{2}$ is shown as a function of the in-plane wave vector $k$ (measured in inverse exciton Bohr radii) for several values of the inverse screening parameter close to the critical value, $1 /\left(q_{s} a^{*}\right)=10$. Solid line: $1 /\left(q_{s} a^{*}\right)=9.9$; dashed line: $1 /\left(q_{s} a^{*}\right)=9.95$; dot-dashed line $1 /\left(q_{s} a\right)=9.98$. Dots show $\delta_{2}(k)$ for $1 /\left(q_{s} a^{*}\right)=10.1$ (a shallow bound state with $m=2$ has just appeared).

the scattering phase shift $\delta_{2}$ is plotted as a function of $k$ for several values of $1 /\left(q_{s} a^{*}\right)$ close to the critical value $1 /\left(q_{s} a^{*}\right)=10$ when the first bound state with $m=2$ appears. One can see that although $\delta_{m}(0)$ has a jump when $q_{s}$ passes a critical value, this jump does not influence the value of the scattering integral if the thermal wave number $q_{T}$ is larger than the interval of $k$ in which $\delta_{m}(k)$ changes rapidly. As shown in Fig. 4, when the bound state disappears the phase shift is affected only in an infinitesimally thin region around $k=0$. For any nonzero temperature this transition region makes no contribution to the phase-shift integral. Thus the electron-hole interaction part of the partition function given by Eq. (16) is a smooth function of the interaction strength, as expected from the general thermodynamic argument. ${ }^{20,40}$ Similar cancellation of the bound-state sum discontinuities for a 3D plasma is well known. ${ }^{41,42}$

The results of the calculation of the two-body interaction part of the partition function for the model semiconductor, ${ }^{13,21}$ for which the assumption $M_{e}=M_{h}$ $=2 \mu_{e h}$ is made, are presented in Fig. 5. Calculations are performed for two values of the ratio of $k_{B} T$ to the bulk excitonic Rydberg, $k_{B} T / \mathrm{Ry}^{*}=1$ (three upper curves) and $k_{B} T / \mathrm{Ry}^{*}=5$ (three lower curves), which roughly correspond to $\mathrm{ZnSe}$ (or $\mathrm{GaN}$ ) and $\mathrm{GaAs}$ at room temperature. Solid lines show the bound-state sum, $Z_{\text {bound }}=\Sigma_{m, \nu} \exp \left(-E_{m, \nu} / k_{B} T\right)$, which exhibits jumps whenever bound states disappear with increasing screening. The electron-hole part of the partition function, $Z_{e h}$, which is shown by dashed lines, is a smooth function of the screening parameter, and the bound-state sum discontinuities are compensated for by the scattering state contributions. Dot-dashed lines show the sum $Z_{e h}+Z_{e e}$, which enters the modified law of mass action [Eq. (4)] (when simplified for the model semiconductor). Note that the cancellation of the $Z_{e h}$ term by the $Z_{e e}$ term for $k_{B} T / \mathrm{Ry} *=5$ is stronger than for $k_{B} T / \mathrm{Ry}^{*}=1$. This can be explained by the enhanced role of scattering states for the higher ratio of $k_{B} T$ to the excitonic Rydberg. The lower absolute value of $Z_{e h}$

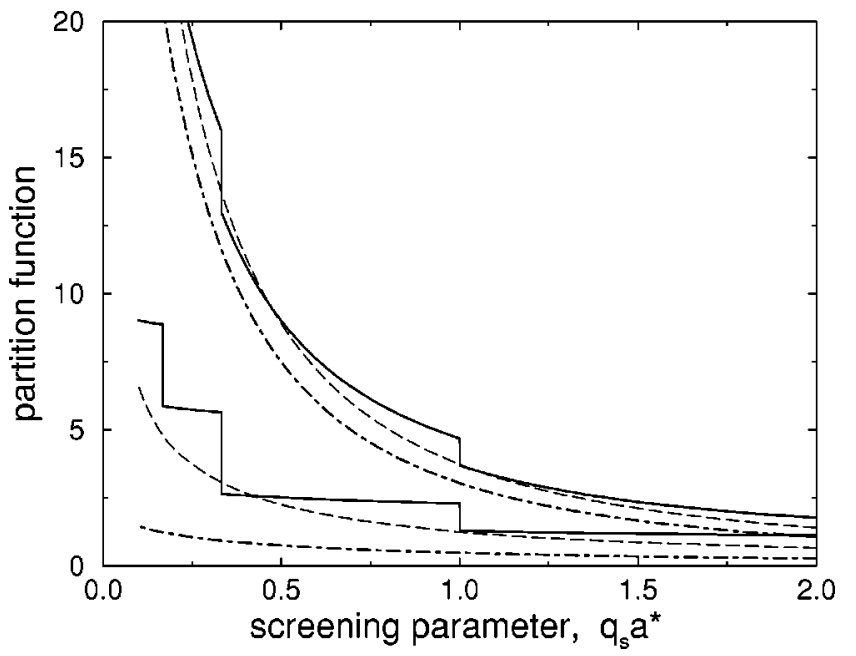

FIG. 5. The two-body interaction part of the partition function vs the inverse screening parameter $1 /\left(q_{s} a^{*}\right)$ for two values of $k_{B} T / \mathrm{Ry}^{*}$. Three upper curves: $k_{B} T=1 \mathrm{Ry} *$; three lower curves: $k_{B} T=5 \mathrm{Ry}^{*}$. Solid lines show the bound state contributions $Z_{\text {bound }}$ only; dashed lines show $Z_{e h}$; dot-dashed lines show $Z_{e h}+Z_{e e}$.

$+Z_{e e}$ ensures that thermodynamic properties of the 2D electron-hole plasma in GaAs are much closer to the ideal gas behavior than those in the case of the wide-gap semiconductor. $^{20}$

For the two-component electron-hole plasma, the Thomas-Fermi 2D screening wave number entering Eq. (7) is given in the Boltzmann limit by ${ }^{29}$

$$
q_{s} a^{*}=\frac{2 \pi \hbar^{2}}{\mu_{e h} k_{B} T}\left(n_{h}^{0}+n_{e}^{0}\right)=4 \pi \frac{\mathrm{Ry}}{k_{B} T}\left(n_{e}^{0} a^{* 2}+n_{h}^{0} a^{* 2}\right) .
$$

Note that we use uncorrelated quasiparticle densities $n_{e}^{0}$ and $n_{h}^{0}$ for the calculation of the screening wave number, since Eq. (17) is derived for the noninteracting $2 \mathrm{D}$ plasma. ${ }^{24,25} \mathrm{We}$ assume that the screening by excitons is much smaller then the free-carrier screening when exciton and free carrier densities are of the same order. However, if one calculates the screening wavenumber using the difference between the total number of carriers and the number of bound carriers, unphysical jumps appear in the dependence of the screening wave number on total density as shallow bound states disappear with increasing density. Thus it is natural to calculate $q_{s}$ on the basis of uncorrelated density $\left(n_{e}^{0}+n_{h}^{0}\right)$, which is a part of the total density behaving as an ideal gas (see Appendix B), and which is a smooth function of the total density.

For the model semiconductor, $n_{h}^{0}=n_{e}^{0}=\alpha n$, and Eq. (17) can be further simplified to

$$
q_{s} a^{*}=8 \pi \alpha \frac{\mathrm{Ry}^{*}}{k_{B} T} n a^{* 2} .
$$

Equation (18) shows clearly the connection between the dimensionless screening parameter $q_{s} a^{*}$ and the two main dimensionless parameters characterizing the 2D electron-hole plasma, namely, the dimensionless density $n a^{* 2}$ and temperature $k_{B} T / \mathrm{Ry}^{*}$. In addition, the role of the degree of ionization, $\alpha$, introduced by Eq. (3) becomes more transparent. The parameter $\alpha$ enters Eq. (18) explicitly, governing the 


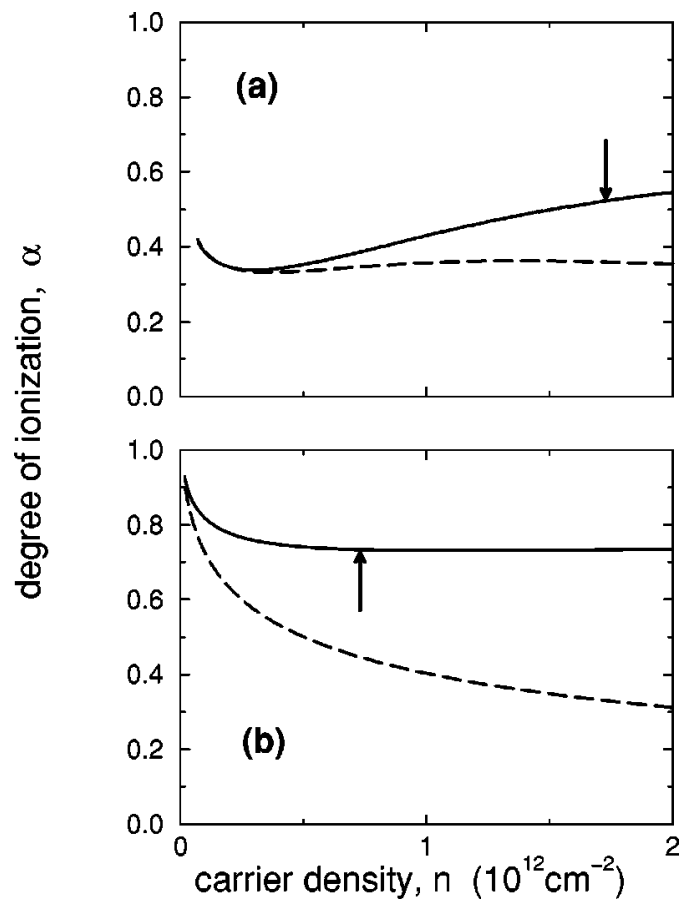

FIG. 6. The degree of ionization (solid lines) of the nondegenerate $2 \mathrm{D}$ electron-hole plasma as a function of the total electron density at room temperature, calculated for the model semiconductor with the effective Bohr radius and excitonic Rydberg of (a) $\mathrm{ZnSe}$, and (b) GaAs. The arrows indicate $n=2 / \lambda_{M_{e}}^{2}$ for $\mathrm{ZnSe}$ and $\mathrm{GaAs}$ at room temperature. Dashed lines show the degree of ionization calculated using a simple law of mass action with a single bound state.

screening wave number which determines the strength of the interaction between charged particles in the plasma. In turn the degree of ionization itself depends on $q_{s} a^{*}$ through the partition functions $Z_{e h}$ and $Z_{e e}$. For the model semiconductor the modified law of mass action [Eq. (4)] can be rewritten as

$$
n_{e}^{\text {corr }} a^{* 2}=4 \pi\left(n_{e}^{0} a^{* 2}\right)^{2} \frac{\mathrm{Ry}}{k_{B} T}\left(Z_{e h}+Z_{e e}\right),
$$

and using Eqs. (3) and (18) we obtain the following expression for the degree of ionization $\alpha$ :

$$
\alpha=\left\{1+\frac{q_{s} a^{*}}{2}\left(Z_{e h}+Z_{e e}\right)\right\}^{-1} .
$$

Equation (20), together with Eqs. (5) and (6) for the partition functions, allows us to calculate the degree of ionization $\alpha$ of the dilute (nondegenerate) 2D electron-hole plasma as a function of the screening parameter $q_{s} a^{*}$. The connection between $q_{s} a^{*}$ and the total electron (hole) density $n$ [Eq. (18)] can be used for self-consistent calculations of $\alpha$ as functions of $n$ for different temperatures or material parameters. The results of these calculations are shown in Fig. 6. Calculations are performed for the model semiconductor with the exciton Bohr radius $a^{*}$ and effective Rydberg Ry* corresponding to ZnSe [Fig. 6(a)] and GaAs [Fig. 6(b)] and for room temperature $\left(k_{B} T=300 \mathrm{~K}\right)$. The arrows indicate the points of crossover from Boltzmann to Fermi statistics, $n=2 / \lambda_{M_{e}}^{2}$. As mentioned in Sec. I, the nondegenerate treatment is more adequate for the wide-gap material.

On the same plot we show, by the dashed lines, the degree of ionization calculated using a simple law of mass action with a single bound state (the ground state of the screened exciton). It can be seen from the figure that the degree of ionization is well described by the single-bound-state mass action law only for low carrier densities. ${ }^{43}$ For high densities (but remaining in the nondegenerate regime) the role of scattering states becomes essential. Instead of the unphysical behavior predicted by the simple mass action law, in which the degree of ionization decreases with increasing density, we find that the degree of ionization increases at higher densities.

A minimum on the curve showing the density dependence of the degree of ionization has the following explanation. At low densities the main contribution to the correlated density comes from the ground exciton state, which is almost unscreened. This state in two dimensions is at least nine times deeper than the first excited state. Therefore, the simple single-bound-state law of mass action is a good approximation at low densities, but not as $n \rightarrow 0-$ when the number of bound states becomes larger than the ground state contribution to the partition function; see Ref. 43. The standard law of mass action states that the density of bound states is proportional to uncorrelated density squared, which reflects the fact that at fixed temperature (room temperature in our case) and low density most of carriers occupy the high-energy ionized states in the continuum rather than the bound states. The low-density high-temperature electron-hole plasma behaves as an ideal gas, with the degree of ionization close to unity. Thus at low density the correlated density is proportional to the square of the total density and the degree of ionization decreases with increasing density. However, with a further increase in the total density, screening becomes important and the inter-particle correlation caused by the Coulomb interaction starts to decrease. Correspondingly, the degree of ionization changes the character of its density dependence. There is a certain value of density, which corresponds to the minimal value of the degree of ionization.

As expected, in wide-gap semiconductors the calculated degree of ionization is much lower than in GaAs for the same temperature and carrier density. For both materials the calculated degree of ionization of the room-temperature 2D electron-hole plasma reaches its minimum at a certain density. The same happens for a $3 \mathrm{D}$ plasma; ${ }^{13,44}$ however, the minimal value of the degree of ionization for the 3D plasma is much higher than in the 2D case (compare Fig. 6 with Fig. 1 in Ref. 44). This is due to the much enhanced binding energy in two dimensions.

The inclusion of Fermi statistics and phase-space filling, which is beyond the scope of the present paper, would provide a sharper rise of $\alpha$ at high carrier densities as the phase space available for the construction of exciton states is restricted. This will apply to both wide-gap and narrow-gap semiconductors. In the foregoing discussion we have assumed a purely $2 \mathrm{D}$ plasma. This assumption gives an overall overestimate of exciton binding energies, compared to a real, finite-width quantum well, for which unscreened exciton binding energies are lower and the finite thickness correction enhances the screening effect. ${ }^{27}$ Thus the results shown in 
Fig. 6 should be considered as lower bound estimates of the degree of ionization of the electron-hole plasmas in $\mathrm{ZnSe}$ and GaAs quantum wells at room temperature. Note that even this lower estimate does not give a value of the degree of ionization of a plasma in a $\mathrm{ZnSe}$ quantum well below 0.33 . This means that at room temperature at least one-third of the carriers are always unbound, which has to be taken into account in gain calculations.

\section{CONCLUSION}

We have calculated the degree of ionization of the 2D electron-hole plasma, taking into account all screened exciton bound states as well as scattering states. It has been shown that the scattering state contribution changes the character of the density dependence of the degree of ionization. We have found that the degree of ionization of the 2D plasma reaches its minimal value at intermediate densities and approaches unity at high densities, which differs from the result based on the simple law of mass action.

The calculated degree of ionization of the electron-hole plasma in a $\mathrm{ZnSe}$ quantum well is significantly lower than in a GaAs quantum well with the same carrier density and temperature. Therefore, excitonic processes should be considered for gain calculations in quantum wells based on widegap semiconductors. However, at room temperature at least one-third of the carriers in $\mathrm{ZnSe}$ wells is shown to be unbound, which allows us to speculate that the most likely lasing mechanism at moderate density is exciton/free-carrier scattering.

Most of the results presented here are obtained for the model system with equal electron and hole effective masses. For wide-gap semiconductors at room temperature $\left(k_{B} T\right.$ $\sim 1 \mathrm{Ry} *$ ) this approximation is good, since $Z_{e e}$ is much smaller than $Z_{e h}$, and the influence of the electron-electron part of the partition function on the degree of ionization is not significant. In the case of an extreme difference between electron and hole masses the model fails, e.g., lighter quasiparticles can be degenerate, when heavy quasiparticles are nondegenerate.

The variable phase method is a powerful tool for studying scattering and bound states in any short-range potential. This method enabled us to find hitherto undiscovered properties of a Coulomb potential statically screened by a 2D electron gas. The same approach can be applied to a more refined potential, which takes into account Friedel oscillations and the finite thickness of the 2D layer.

\section{ACKNOWLEDGMENTS}

This work was supported by the UK EPSRC and the Royal Society, and we thank Dr. S.-C. Lee and H. Ouerdane for a careful reading of the manuscript.

\section{APPENDIX A: VARIABLE PHASE METHOD IN TWO DIMENSIONS}

In this appendix we derive the basic equations of the variable phase approach in two dimensions from the radial Schrödinger equation. This derivation is similar to that in three dimensions. ${ }^{22}$

\section{Scattering phase shifts}

The relative in-plane motion of two interacting particles with masses $M_{a}$ and $M_{b}$ and the energy of relative motion $E$ can be considered as a motion of a particle with the mass $\mu_{a b}=M_{a} M_{b} /\left(M_{a}+M_{b}\right)$ and energy $E$, moving in an external central potential $V(\rho)$. This motion is described by the wave function satisfying the stationary Schrödinger equation

$$
\hat{H}_{r e l} \psi=-\frac{\hbar^{2}}{2 \mu_{a b}}\left(\frac{1}{\rho} \frac{\partial}{\partial \rho} \rho \frac{\partial}{\partial \rho}+\frac{1}{\rho^{2}} \frac{\partial^{2}}{\partial \varphi^{2}}\right) \psi+V(\rho) \psi=E \psi .
$$

Owing to the axial symmetry of the potential $V(\rho)$, we can separate variables in the expression for the wave function

$$
\psi_{m}(\rho, \varphi)=R_{m}(\rho) e^{i m \varphi}, \quad m=0, \pm 1, \pm 2, \ldots
$$

The equation for the radial function $R_{m}(\rho)$ reads

$$
R_{m}^{\prime \prime}+\frac{1}{\rho} R_{m}^{\prime}+\left(k^{2}-U(\rho)-\frac{m^{2}}{\rho^{2}}\right) R_{m}=0,
$$

where $k^{2}=2 \mu_{a b} E / \hbar^{2}$ and $U(\rho)=2 \mu_{a b} V(\rho) / \hbar^{2}$. In what follows we consider $m \geqslant 0$ only, as $R_{-m}(\rho)=R_{m}(\rho)$.

We assume that the interaction potential vanishes at infinity (the precise decay rate will be discussed later). Then at large distances the radial function satisfies the free Bessel equation, whose general solution is

$$
\begin{aligned}
R_{m}(\rho) & =A_{m}\left[J_{m}(k \rho) \cos \delta_{m}-N_{m}(k \rho) \sin \delta_{m}\right] \\
& \stackrel{\rho \rightarrow \infty}{\rightarrow} A_{m}\left(\frac{2}{\pi k \rho}\right)^{1 / 2} \cos \left[k \rho-(2 m+1) \pi / 4+\delta_{m}\right],
\end{aligned}
$$

where $\delta_{m}$ is the scattering phase shift, ${ }^{17,18}$ and $J_{m}(k \rho)$ and $N_{m}(k \rho)$ are the Bessel and the Neumann functions, respectively.

In the variable phase approach, $A_{m}$ and $\delta_{m}$ are considered not as constants but as functions of the distance $\rho$. The amplitude function $A_{m}(\rho)$ and the phase function $\delta_{m}(\rho)$ are introduced by the equation

$$
R_{m}(\rho)=A_{m}(\rho)\left[J_{m}(k \rho) \cos \delta_{m}(\rho)-N_{m}(k \rho) \sin \delta_{m}(\rho)\right],
$$

with the additional condition, which we are free to choose as

$$
R_{m}^{\prime}(\rho)=A_{m}(\rho)\left[J_{m}^{\prime}(k \rho) \cos \delta_{m}(\rho)-N_{m}^{\prime}(k \rho) \sin \delta_{m}(\rho)\right],
$$

where the prime indicates differentiation with respect to $\rho$. The phase function $\delta_{m}(\rho)$ has a natural physical interpretation as being the phase shift produced by a potential cut off at a distance $\rho$.

Differentiating Eq. (A6) and substituting the resulting expression, together with Eqs. (A5) and (A6), into Eq. (A3), we obtain 


$$
\begin{aligned}
A_{m}^{\prime}(\rho)[ & \left.J_{m}^{\prime}(k \rho) \cos \delta_{m}(\rho)-N_{m}^{\prime}(k \rho) \sin \delta_{m}(\rho)\right]-\delta_{m}^{\prime}(\rho) A_{m}(\rho) \\
\times & {\left[J_{m}^{\prime}(k \rho) \sin \delta_{m}(\rho)+N_{m}^{\prime}(k \rho) \cos \delta_{m}(\rho)\right] } \\
= & U(\rho) A_{m}(\rho)\left[J_{m}(k \rho) \cos \delta_{m}(\rho)\right. \\
& \left.-N_{m}(k \rho) \sin \delta_{m}(\rho)\right] .
\end{aligned}
$$

To obtain Eq. (A7) we used the fact that the functions $J_{m}(k \rho)$ and $N_{m}(k \rho)$ satisfy the free Bessel equation

$$
F_{m}^{\prime \prime}+\frac{1}{\rho} F_{m}^{\prime}+\left(k^{2}-\frac{m^{2}}{\rho^{2}}\right) F_{m}=0 .
$$

Equating the derivative of Eq. (A5) to Eq. (A6) implies the following condition on the derivatives of the amplitude and the phase functions:

$$
\begin{aligned}
& A_{m}^{\prime}(\rho)\left[J_{m}(k \rho) \cos \delta_{m}(\rho)-N_{m}(k \rho) \sin \delta_{m}(\rho)\right] \\
& \quad=\delta_{m}^{\prime}(\rho) A_{m}(\rho)\left[J_{m}(k \rho) \sin \delta_{m}(\rho)+N_{m}(k \rho) \cos \delta_{m}(\rho)\right] .
\end{aligned}
$$

Substituting $A_{m}^{\prime}(\rho)$, obtained from Eq. (A8), into Eq. (A7) yields

$$
\begin{aligned}
& -\delta_{m}^{\prime}(\rho)\left[J_{m}(k \rho) N_{m}^{\prime}(k \rho)-N_{m}(k \rho) J_{m}^{\prime}(k \rho)\right] \\
& \quad=U(\rho)\left[J_{m}(k \rho) \cos \delta_{m}(\rho)-N_{m}(k \rho) \sin \delta_{m}(\rho)\right]^{2} .
\end{aligned}
$$

Equation (A9) can be simplified further, using the Wronskian of the Bessel functions,

$$
\begin{aligned}
W\left\{J_{m}(x), N_{m}(x)\right\} & =J_{m}(x) \frac{d}{d x} N_{m}(x)-N_{m}(x) \frac{d}{d x} J_{m}(x) \\
& =\frac{2}{\pi x},
\end{aligned}
$$

and thus becomes

$$
\begin{aligned}
\frac{d}{d \rho} \delta_{m}(\rho)= & -\frac{\pi}{2} \rho U(\rho)\left[J_{m}(k \rho) \cos \delta_{m}(\rho)\right. \\
& \left.-N_{m}(k \rho) \sin \delta_{m}(\rho)\right]^{2} .
\end{aligned}
$$

This phase equation, Eq. (A10), is a first-order, nonlinear differential equation of the Ricatti type, which must be solved with the initial condition

$$
\delta_{m}(0)=0,
$$

thus ensuring that the radial function does not diverge at $\rho$ $=0$. The total scattering phase shift $\delta_{m}$ can be obtained as a large-distance limit of the phase function $\delta_{m}(\rho)$ :

$$
\delta_{m}=\lim _{\rho \rightarrow \infty} \delta_{m}(\rho) .
$$

For numerical convenience, instead of the initial condition Eq. (A11), the small- $\rho$ expansion is used:

$$
\delta_{m}(\rho) \approx-\frac{\pi k^{2 m}}{2^{2 m+1}(m !)^{2}} \int_{0}^{\rho} U\left(\rho^{\prime}\right) \rho^{\prime 2 m+1} d \rho^{\prime}, \quad \rho \rightarrow 0 .
$$

From Eq. (A10) and the asymptotic expansions of the Bessel functions one can see that the variable phase method is applicable only if the scattering potential $U(\rho)$ satisfies the necessary conditions

$$
\int_{\rho}^{\infty} U\left(\rho^{\prime}\right) d \rho^{\prime} \rightarrow 0, \quad \rho \rightarrow \infty
$$

and

$$
\rho^{2} U(\rho) \rightarrow 0, \quad \rho \rightarrow 0 .
$$

The statically screened Coulomb potential $V_{s}(\rho)$, defined by Eq. (7), behaves like $\rho^{-1}$ at small distances and like $\rho^{-3}$ at large distances. Such behavior allows the application of the variable phase method to this potential.

\section{Bound-state energies}

For the states with negative energy of the relative motion (bound states), the wave number $k$ is imaginary, $k=i \kappa$, and we introduce the function $\eta_{m}(\rho, \kappa)$ vanishing in the origin and satisfying a nonlinear equation

$$
\begin{aligned}
\frac{d}{d \rho} \eta_{m}(\rho, \kappa)= & -\frac{\pi}{2} \rho U(\rho)\left[I_{m}(\kappa \rho) \cos \eta_{m}(\rho, \kappa)\right. \\
& \left.+\frac{2}{\pi} K_{m}(\kappa \rho) \sin \eta_{m}(\rho, \kappa)\right]^{2},
\end{aligned}
$$

where $I_{m}(\kappa \rho)$ and $K_{m}(\kappa \rho)$ are the modified Bessel functions of the first and second kinds, respectively. Equation (A16) is derived in the same fashion as Eq. (A10). The functions $I_{m}(\kappa \rho)$ and $K_{m}(\kappa \rho)$ represent two linearly independent solutions of the free radial-wave Schrödinger equation for the negative value of energy, $E=-\hbar^{2} \kappa^{2} / 2 \mu_{a b}$, and $\cot \eta_{m}$ characterizes the weights of the diverging $\left[I_{m}(\kappa \rho)\right]$ and converging $\left[K_{m}(\kappa \rho)\right]$ solutions as $\rho \rightarrow \infty$. For the bound state, the diverging solution vanishes, implying the asymptotic condition

$$
\eta_{m}\left(\rho \rightarrow \infty, \kappa_{\nu}\right)=(\nu-1 / 2) \pi, \nu=1,2, \ldots
$$

Here $\nu$ numerates the bound states for a given $m$, and ( $\nu$ -1 ) is the number of non-zero nodes of the radial wave function. For numerical solution of Eq. (A16), instead of the boundary condition $\eta_{m}(0, \kappa)=0$, an asymptotic initial condition [analogous to the condition Eq. (A13) for the phase function $\left.\delta_{m}(\rho)\right]$ is used.

\section{APPENDIX B: BETH-UHLENBECK FORMULA IN TWO DIMENSIONS}

In this appendix we derive Eq. (5), which is the 2D analog of the Beth-Uhlenbeck formula, ${ }^{19}$ and the modified law of the mass action [Eq. (4)]. This derivation is similar to the analysis used for the calculation of the second virial coefficient of low-density ${ }^{3} \mathrm{He}$ and ${ }^{4} \mathrm{He}$ monolayers on graphite. ${ }^{45}$

Let us consider a binary mixture of components $a$ and $b$ 
in two dimensions. The grand partition function of the mixture is given by

$$
\Omega\left(z_{a}, z_{b}, A, T\right)=\sum_{N_{a}, N_{b}} \mathcal{Q}_{N_{a}, N_{b}} z_{a}^{N_{a}} z_{b}^{N_{b}}
$$

where $z_{a}$ and $z_{b}$ are the fugacities $\left(z_{a}=e^{\beta \mu_{a}}\right.$, with $\mu_{a}$ being the chemical potential of the component $a$ ), $A$ is the area of the $2 \mathrm{D}$ system, and $\mathcal{Q}_{N_{a}, N_{b}}$ is the partition function defined as

$$
\mathcal{Q}_{N_{a}, N_{b}}(A, T)=\operatorname{Tr} e^{-\beta \hat{H}\left(N_{a}, N_{b}\right)},
$$

where the trace is to be taken over all states of the system that has $N_{a}$ particles of the type $a$ and $N_{b}$ particles of the type $b$ in the area $A$.

We now expand the quantity $\ln \Omega$ as a power series in $z_{a}$ and $z_{b}$ :

$$
\ln \Omega\left(z_{a}, z_{b}, A, T\right)=\sum_{l_{a}, l_{b}} A \mathcal{C}_{l_{a}, l_{b}} z_{a}^{l_{a}} z_{b}^{l_{b}}
$$

The density of the component $a$ is given by

$$
n_{a}=\frac{1}{A} z_{a} \frac{d}{d z_{a}} \ln \Omega=\sum_{l_{a}, l_{b}} l_{a} \mathcal{C}_{l_{a}, l_{b}} z_{a}^{l_{a}} z_{b}^{l_{b}} .
$$

From this point we consider the low-density limit, $z_{a}, z_{b}$ $\ll 1$, and neglect all the terms higher than $z^{2}$ in Eq. (B4). Then

$$
n_{a} \approx \mathcal{C}_{1,0} z_{a}+\mathcal{C}_{1,1} z_{a} z_{b}+2 \mathcal{C}_{2,0} z_{a}^{2}
$$

From comparing corresponding powers in Eqs. (B3) and (B1), we obtain

$$
\begin{gathered}
\mathcal{C}_{1,0}=\mathcal{Q}_{1,0} / A, \\
\mathcal{C}_{1,1}=\left(\mathcal{Q}_{1,1}-\mathcal{Q}_{1,0} \mathcal{Q}_{0,1}\right) / A,
\end{gathered}
$$

and

$$
\mathcal{C}_{2,0}=\left(\mathcal{Q}_{2,0}-\frac{1}{2} \mathcal{Q}_{1,0}^{2}\right) / A
$$

The next step is to calculate the partition functions entering Eqs. (B6)-(B8). First of all, the one-particle partition function $\mathcal{Q}_{1,0}$ is given by

$$
\mathcal{Q}_{1,0}(A, T)=g_{a} \frac{A}{(2 \pi)^{2}} \int d^{2} \mathbf{k} \exp \left(-\beta \frac{\hbar^{2} k^{2}}{2 M_{a}}\right)=g_{a} \frac{A}{\lambda_{M_{a}}^{2}},
$$

where $g_{a}$ is a quantum state degeneracy and $\lambda_{M_{a}}$ is a thermal wavelength,

$$
\lambda_{M_{a}}^{2}=\frac{2 \pi \beta \hbar^{2}}{M_{a}} .
$$

This yields

$$
\mathcal{C}_{1,0}=\frac{g_{a}}{\lambda_{M_{a}}^{2}}
$$

In order to find the two-particle partition function $\mathcal{Q}_{1,1}$ it is useful to separate the center-of-mass motion and the relative motion of the two particles:

$$
\begin{aligned}
\mathcal{Q}_{1,1}(A, T) & =g_{a} g_{b} \frac{A}{\lambda_{M_{a}+M_{b}}^{2}} \operatorname{Tr} e^{-\beta \hat{H}_{r e l}} \\
& =g_{a} g_{b} \frac{A}{\lambda_{M_{a}+M_{b}}^{2}} \int d^{2} \boldsymbol{\rho} \sum_{n}\left|\psi_{n}(\boldsymbol{\rho})\right|^{2} e^{-\beta E_{n},}
\end{aligned}
$$

where the factor $A / \lambda_{M_{a}+M_{b}}^{2}$ appears from performing the summation over all center-of-mass momenta, the Hamiltonian $\hat{H}_{\text {rel }}$ of the relative motion is given in Appendix A, and the sum in Eq. (B12) is taken over all different solutions of Eq. (A1).

For the corresponding two-body system of noninteracting distinguishable particles, one would have

$$
\mathcal{Q}_{1,1}^{(0)}(A, T)=g_{a} g_{b} \frac{A}{\lambda_{M_{a}+M_{b}}^{2}} \int d^{2} \boldsymbol{\rho} \sum_{n}\left|\psi_{n}^{(0)}(\boldsymbol{\rho})\right|^{2} e^{-\beta E_{n}^{(0)}},
$$

where the superscript (0) refers to quantities of the noninteracting system. The two-body interaction part of the partition function is then defined by

$$
\begin{aligned}
Z_{a b} & =\int d^{2} \boldsymbol{\rho} \sum_{n}\left\{\left|\psi_{n}(\boldsymbol{\rho})\right|^{2} e^{-\beta E_{n}}-\left|\psi_{n}^{(0)}(\boldsymbol{\rho})\right|^{2} e^{-\beta E_{n}^{(0)}}\right\} \\
& =\sum_{n}\left\{e^{-\beta E_{n}}-e^{-\beta E_{n}^{(0)}}\right\} .
\end{aligned}
$$

Thus

$$
\mathcal{Q}_{1,1}(A, T)=\mathcal{Q}_{1,1}^{(0)}(A, T)+g_{a} g_{b} \frac{A}{\lambda_{M_{a}+M_{b}}^{2}} Z_{a b} .
$$

To analyze Eq. (B14) further, we must study the energy spectra $E_{n}^{(0)}$ and $E_{n}$. For the noninteracting system, $E_{n}^{(0)}$ forms a continuum. We write

$$
E_{n}^{(0)}=\frac{\hbar^{2} k^{2}}{2 \mu_{a b}},
$$

which defines the relative wave number $k$. Then for the system of two noninteracting distinguishable particles the function $\mathcal{Q}_{1,1}^{(0)}$ given by Eq. (B13) can be easily evaluated as

$$
\begin{aligned}
\mathcal{Q}_{1,1}^{(0)}(A, T) & =g_{a} g_{b} \frac{A}{\lambda_{M_{a}+M_{b}}^{2}} \frac{A}{(2 \pi)^{2}} \int d^{2} \mathbf{k} \exp \left(-\beta \frac{\hbar^{2} k^{2}}{2 \mu_{a b}}\right) \\
& =g_{a} g_{b} \frac{A}{\lambda_{M_{a}+M_{b}}^{2}} \frac{A}{\lambda_{\mu_{a b}}^{2}}=\left(g_{a} \frac{A}{\lambda_{M_{a}}^{2}}\right)\left(g_{b} \frac{A}{\lambda_{M_{b}}^{2}}\right) \\
& =\mathcal{Q}_{1,0} \mathcal{Q}_{0,1} .
\end{aligned}
$$


For the interacting system, the spectrum of $E_{n}$ in general contains a discrete set of values $E_{B}$, corresponding to twobody bound states, and a continuum. In the continuum, we define the wave number $k$ for the interacting system by putting

$$
E_{n}=\frac{\hbar^{2} k^{2}}{2 \mu_{a b}}
$$

Let $g(k) d k$ be the number of states with a wave number lying between $k$ and $k+d k$, and let $g^{(0)}(k) d k$ denote the corresponding quantity for the noninteracting system. Then Eq. (B14) can be written in the form

$$
Z_{a b}=\sum_{B} e^{-\beta E_{B}}+\int_{0}^{\infty} d k\left\{g(k)-g^{(0)}(k)\right\} \exp \left(-\beta \frac{\hbar^{2} k^{2}}{2 \mu_{a b}}\right) .
$$

The difference in density of states is related to the scattering phase shifts by the following argument. ${ }^{45}$ The relative wave function can be factorized [see Eq. (A2)] into a product of a trivial azimuthal part and nontrivial radial wave function $R_{m}(\rho)$, which satisfies Eq. (A3). For large value of $\rho$ where the potential is assumed negligible,

$$
R_{m}(\rho \rightarrow \infty) \propto \cos \left\{k \rho-(2 m+1) \pi / 4+\delta_{m}(k)\right\},
$$

which defines the phase shift $\delta_{m}(k)$ of the $m$ th partial wave. For the noninteracting system all the phase shifts $\delta_{m}(k) \equiv 0$. If the system is placed within a circle of radius $R$, the vanishing of the wave function at the boundary requires that the allowed values of $k$ are given by

$$
k R-(2 m+1) \pi / 4+\delta_{m}(k)=\left(n+\frac{1}{2}\right) \pi
$$

for the interacting system, and

$$
k R-(2 m+1) \pi / 4=\left(n+\frac{1}{2}\right) \pi
$$

for the noninteracting system, where $n=0,1,2, \ldots$ For a given $m$, changing $n$ by one unit causes $k$ to change by the respective amounts $\Delta k$ and $\Delta k^{(0)}$ :

$$
\begin{gathered}
\Delta k=\frac{\pi}{R+\left[d \delta_{m}(k) / d k\right]}, \\
\Delta k^{(0)}=\frac{\pi}{R} .
\end{gathered}
$$

These are the spacings of eigenvalues for a given $m$. Let the number of states of a given $m$ with wave number lying between $k$ and $k+d k$ be denoted by $g_{m}(k) d k$ and $g_{m}^{(0)}(k) d k$ for the two cases. We must have

$$
\begin{aligned}
& g_{m}(k) \Delta k=1, \\
& g_{m}^{(0)}(k) \Delta k=1
\end{aligned}
$$

or

$$
g_{m}(k)=\frac{1}{\pi}\left(R+\frac{d \delta_{m}(k)}{d k}\right),
$$

$$
g_{m}^{(0)}(k)=\frac{1}{\pi} R
$$

Therefore,

$$
g_{m}(k)-g_{m}^{(0)}(k)=\frac{1}{\pi} \frac{d \delta_{m}(k)}{d k} .
$$

Summing Eq. (B29) over all allowed $m$, we obtain

$$
g(k)-g^{(0)}(k)=\frac{1}{\pi} \sum_{m} \frac{d \delta_{m}(k)}{d k} .
$$

Substituting Eq. (B30) into Eq. (B19) yields

$$
\begin{aligned}
Z_{e h}= & \sum_{B} e^{-\beta E_{B}}+\frac{1}{\pi} \int_{0}^{\infty}\left(\sum_{m=-\infty}^{\infty} \frac{d \delta_{m}(k)}{d k}\right) \\
& \times \exp \left(-\beta \frac{\hbar^{2} k^{2}}{2 \mu_{a b}}\right) d k,
\end{aligned}
$$

which coincides with Eq. (5) if we change the notation for the bound-state energy from $E_{B}$ to $E_{m, \nu}$, where subscript $\nu$ enumerates bound states with a given $m$.

Now, having evaluated the interaction part of the partition function, we can obtain the coefficient $\mathcal{C}_{1,1}$ needed in the density expansion, by substituting Eq. (B15) into Eq. (B7) and taking into account Eq. (B17):

$$
\mathcal{C}_{1,1}=g_{a} g_{b} \frac{1}{\lambda_{M_{a}+M_{b}}^{2}} Z_{a b}=\lambda_{\mu_{a b}}^{2} \frac{g_{a}}{\lambda_{M_{a}}^{2}} \frac{g_{b}}{\lambda_{M_{b}}^{2}} Z_{a b}
$$

Up to this point we have considered a system of two distinguishable particles (e.g., an electron and a hole). To calculate the remaining coefficient $\mathcal{C}_{2,0}$ in Eq. (B5), we must now consider the interaction of indistinguishable particles. For such particles the sum over different $m$ is modified, e.g., for two spinless bosons the relative wave function must be symmetric; this means that only even values of $m$ are possible. For the case of two fermions with the same spin the coordinate wave function must by antisymmetric. Let us consider a case of the two fermions or bosons of the same type $a$, with the quantum state degeneracy $g_{a}$. This degeneracy is usually associated with the particle spin $s$, so that $g_{a}=2 s$ +1 . For a two-particle system there are $g_{a}^{2}=(2 s+1)^{2}$ spin states, of which a fraction $(s+1) /(2 s+1)=\left(g_{a}+1\right) /\left(2 g_{a}\right)$ are symmetric and a fraction $s /(2 s+1)=\left(g_{a}-1\right) /\left(2 g_{a}\right)$ are antisymmetric. For a fermion system, the symmetric spin states must be multiplied by antisymmetric spatial states (odd $\mathrm{m}$ ) and the antisymmetric spin states multiply symmetric spatial states (even $m$ ). A similar argument is applicable for bosons. Thus, for the 2D system of interacting particles of the type $a$, the two-body partition function is

$$
\mathcal{Q}_{2,0}(A, T)=\mathcal{Q}_{2,0}^{(0)}(A, T)+g_{a}^{2} \frac{A}{\lambda_{M_{a}}^{2}} Z_{a a},
$$

where $Z_{a a}$ is defined by 


$$
\begin{aligned}
Z_{a a}= & \sum_{m, \nu} \frac{g_{a} \pm(-1)^{m}}{g_{a}} e^{-\beta E_{m, \nu}} \\
& +\frac{1}{\pi} \sum_{m=-\infty}^{\infty} \frac{g_{a} \pm(-1)^{m}}{g_{a}} \int_{0}^{\infty} \frac{d \delta_{m}(k)}{d k} \exp \left(-\beta \frac{\hbar^{2} k^{2}}{M_{a}}\right) d k,
\end{aligned}
$$

Here and in what follows the upper sign is for bosons and the lower sign is for fermions. For the case of repulsive fermions (which do not form bound states) and for $g_{a}=2$, Eq. (B34) reduces to Eq. (6).

Let us now calculate the two-body partition function of noninteracting bosons or fermions, $\mathcal{Q}_{2,0}^{(0)}$, which is given by

$$
\begin{aligned}
\mathcal{Q}_{2,0}^{(0)}(A, T)= & \frac{A}{\lambda_{2 M_{a}}^{2}} \frac{g_{a}}{2} \int d^{2} \boldsymbol{\rho} \sum_{\mathbf{k}}{ }^{\prime}\left\{\left(g_{a} \pm 1\right)\left|\psi_{s}^{(0)}(\mathbf{k}, \boldsymbol{\rho})\right|^{2}\right. \\
& \left.+\left(g_{a} \mp 1\right)\left|\psi_{a}^{(0)}(\mathbf{k}, \boldsymbol{\rho})\right|^{2}\right\} \exp \left(-\beta \frac{\hbar^{2} k^{2}}{M_{a}}\right),
\end{aligned}
$$

where $\psi_{s}^{(0)}(\mathbf{k}, \boldsymbol{\rho})$ and $\psi_{a}^{(0)}(\mathbf{k}, \boldsymbol{\rho})$ are symmetric and antisymmetric eigenfunctions of the noninteracting Hamiltonian of the relative motion, i.e.,

$$
\psi_{s}^{(0)}(\mathbf{k}, \boldsymbol{\rho})=\sqrt{\frac{2}{A}} \cos (\mathbf{k} \cdot \boldsymbol{\rho})
$$

and

$$
\psi_{a}^{(0)}(\mathbf{k}, \boldsymbol{\rho})=\sqrt{\frac{2}{A}} \sin (\mathbf{k} \cdot \boldsymbol{\rho})
$$

The summation in Eq. (B35) must be performed over all different two-particle states. Wave vectors $\mathbf{k}$ and $\mathbf{- k}$ correspond to the same state ( $\mathbf{k}$ and $-\mathbf{k}$ transfer into each other upon exchange of the two indistinguishable particles), this state should not be counted twice, and

$$
\sum_{\mathbf{k}}^{\prime} \rightarrow \frac{1}{2} \frac{A}{(2 \pi)^{2}} \int d^{2} \mathbf{k}
$$

Then $\mathcal{Q}_{2,0}^{(0)}$ can be written as

$$
\mathcal{Q}_{2,0}^{(0)}(A, T)=g_{a} \frac{A}{\lambda_{M_{a}}^{2}}\left\{\left(g_{a} \pm 1\right) \mathcal{I}_{s}+\left(g_{a} \mp 1\right) \mathcal{I}_{a}\right\},
$$

where

$$
\begin{aligned}
\mathcal{I}_{s} & =\frac{1}{(2 \pi)^{2}} \int d^{2} \boldsymbol{\rho} d^{2} \mathbf{k} \cos ^{2}(\mathbf{k} \cdot \boldsymbol{\rho}) \exp \left(-\beta \frac{\hbar^{2} k^{2}}{M_{a}}\right) \\
& =\frac{1}{2}\left(\frac{A}{2 \lambda_{M_{a}}^{2}}+\frac{1}{4}\right)
\end{aligned}
$$

and

$$
\begin{aligned}
\mathcal{I}_{a} & =\frac{1}{(2 \pi)^{2}} \int d^{2} \boldsymbol{\rho} d^{2} \mathbf{k} \sin ^{2}(\mathbf{k} \cdot \boldsymbol{\rho}) \exp \left(-\beta \frac{\hbar^{2} k^{2}}{M_{a}}\right) \\
& =\frac{1}{2}\left(\frac{A}{2 \lambda_{M_{a}}^{2}}-\frac{1}{4}\right) .
\end{aligned}
$$

Finally,

$$
\mathcal{Q}_{2,0}^{(0)}(A, T)=\frac{1}{2}\left(g_{a} \frac{A}{\lambda_{M_{a}}^{2}}\right)^{2} \pm \frac{1}{4} g_{a} \frac{A}{\lambda_{M_{a}}^{2}}=\frac{1}{2} \mathcal{Q}_{1,0}^{2} \pm \frac{1}{4} g_{a} \frac{A}{\lambda_{M_{a}}^{2}} .
$$

Substituting Eqs. (B33) and (B42) into Eq. (B8) yields

$$
\mathcal{C}_{2,0}=\frac{1}{2} \lambda_{\mu_{a a}}^{2}\left(\frac{g_{a}}{\lambda_{M_{a}}^{2}}\right)^{2} Z_{a a} \pm \frac{1}{4} \frac{g_{a}}{\lambda_{M_{a}}^{2}}
$$

Substituting Eqs. (B11), (B32), and (B43) into Eq. (B5), we find the following expression for the total density of the component $a$ :

$$
\begin{aligned}
n_{a} \approx & \frac{g_{a}}{\lambda_{M_{a}}^{2}} z_{a} \pm \frac{1}{2} \frac{g_{a}}{\lambda_{M_{a}}^{2}} z_{a}^{2}+\lambda_{\mu_{a a}}^{2}\left(\frac{g_{a}}{\lambda_{M_{a}}^{2}}\right)^{2} Z_{a a} z_{a}^{2} \\
& +\lambda_{\mu_{a b}}^{2} \frac{g_{a}}{\lambda_{M_{a}}^{2}} \frac{g_{b}}{\lambda_{M_{b}}^{2}} Z_{a b} z_{a} z_{b} .
\end{aligned}
$$

The first two terms in Eq. (B44) do not depend on interparticle interaction (however, the second term depends via its sign on the statistics of the particles). It is natural to call the sum of two first terms in Eq. (B44) an uncorrelated density; we denote it as $n_{a}^{0}$. Note that these terms are the first two terms in the low-density expansion of the well-known expression $^{25}$ for the density of the noninteracting 2D Bose or Fermi gases:

$$
n_{a}^{0}=\mp \frac{g_{a}}{\lambda_{M_{a}}^{2}} \ln \left(1 \mp z_{a}\right) .
$$

The sum of the last two terms in Eq. (B44) is the interactiondependent correlated density $n_{a}^{\text {corr }}$.

In the low-density limit the second term in Eq. (B44) is much smaller than the first one, and within the same accuracy as Eq. (B44) we can write

$$
n_{a}^{\text {corr }} \approx \sum_{b} n_{a}^{0} n_{b}^{0} \lambda_{\mu_{a b}^{2}} Z_{a b}
$$

Equation (B45) coincides with Eq. (4), and it constitutes the modified law of the mass action in two dimensions.
*Present address: School of Physics, University of Exeter, Exeter EX4 4QL, United Kingdom; also at A. F. Ioffe PhysicalTechnical Institute, St. Petersburg, Russia.

${ }^{1}$ S. Taniguchi, T. Hino, S. Itoh, K. Nakano, N. Nakayama, A.
Ishibashi, and M. Ikeda, Electron. Lett. 32, 552 (1996).

${ }^{2}$ S. Nakamura, M. Senoh, S. Nagahama, N. Iwasa, T. Yamada, T. Matsushita, Y. Sugimoto, and H. Kiyoku, Appl. Phys. Lett. 70, 1417 (1997). 
${ }^{3}$ H. Haug and S.W. Koch, Phys. Status Solidi B 82, 531 (1977); S.W. Koch, H. Haug, G. Schmeider, W. Bohnert, and C. Klingshirn, ibid. 89, 431 (1978)

${ }^{4}$ W.W. Chow, S.W. Koch, and M. Sargent III, SemiconductorLaser Physics (Springer, Berlin, 1994).

${ }^{5}$ E. Hanamura and H. Haug, Phys. Rep. 33C, 209 (1977).

${ }^{6}$ M. Lindberg, Y.Z. Hu, R. Binder, and S.W. Koch, Phys. Rev. B 50, 18060 (1994).

${ }^{7}$ M.F. Pereira, Jr. and K. Henneberger, Phys. Rev. B 58, 2064 (1998).

${ }^{8}$ T.M. Rice, Solid State Phys. 32, 1 (1977).

${ }^{9}$ S. Schmitt-Rink, D.S. Chemla, and D.A.B. Miller, Adv. Phys. 38, 89 (1989).

${ }^{10}$ J. Ding, M. Hagerott, T. Ishihara, H. Jeon, and A.V. Nurmikko, Phys. Rev. B 47, 10528 (1993)

${ }^{11}$ M. Schmidt, G. Röpke, and H. Schultz, Ann. Phys. (N.Y.) 202, 57 (1990); G. Röpke, A. Schnell, P. Schuck, and P. Nozières, Phys. Rev. Lett. 80, 3177 (1998).

${ }^{12}$ W.-D. Kraeft, D. Kremp, W. Ebeling, and G. Röpke, Quantum Statistics of Charged Particle Systems (Akademie-Verlag, Berlin, 1986).

${ }^{13}$ R. Zimmermann and H. Stolz, Phys. Status Solidi B 131, 151 (1985); R. Zimmermann, Many-Particle Theory of Highly Excited Semiconductors (Teubner, Leipzig, 1987).

${ }^{14}$ I. Galbraith, in Microscopic Theory of Semiconductors: Quantum Kinetics, Confinement and Lasers, edited by S. W. Koch (World Scientific, Singapore, 1996), p. 211, and references therein.

${ }^{15}$ R. Cingolani, L. Calcagnile, G. Colí, R. Rinaldi, M. Lomascolo, M. DiDio, A. Franciosi, L. Vanzetti, G.C. LaRocca, and D. Campi, J. Opt. Soc. Am. B 13, 1268 (1996); R. Cingolani, G. Colí, L. Calcagnile, R. Rinaldi, A.L. Convertino, M. Lomascolo, M. DiDio, I. Suemune, Phys. Rev. B 54, 17812 (1996).

${ }^{16}$ N. Ben Brahim Aouani, L. Mandhour, R. Bennaceur, S. Jasiri, T. Amand, and X. Marie, Solid State Commun. 108, 199 (1998).

${ }^{17}$ F. Stern and W.E. Howard, Phys. Rev. 163, 816 (1967).

${ }^{18}$ L.D. Landau and E.M. Lifshitz, Quantum Mechanics (NonRelativistic Theory) (Butterworth-Heinemann, Oxford, 1997), p. 508.

${ }^{19}$ E. Beth and G.E. Uhlenbeck, Physica (Amsterdam) 4, 915 (1937); see also, e.g., L.D. Landau and E.M. Lifshitz, Statistical Physics (Pergamon, New York, 1980), Pt. 1, p. 236.

${ }^{20}$ M.E. Portnoi and I. Galbraith, Phys. Rev. B 58, 3963 (1998).

${ }^{21}$ P. Vashishta and R.K. Kalia, Phys. Rev. B 25, 6492 (1982); X. Zhu, M.S. Hybertsen, and P.B. Littlewood, ibid. 54, 13575 (1996).

${ }^{22}$ F. Calogero, Variable Phase Approach to Potential Scattering (Academic, New York, 1967).

${ }^{23}$ T. Ando, A.B. Fowler, and F. Stern, Rev. Mod. Phys. 54, 437 (1982); for a more recent development see, e.g., C. Bulutay and M. Tomak, Phys. Rev. B 54, 14643 (1996); K. Schrüfer, C. Metzner, M.Ch. Hofmann, and G.H. Döhler, Superlattices Mi- crostruct. 21, 223 (1997); A. Gold and A. Ghazali, J. Phys.: Condens. Matter 9, 6885 (1997), and references therein.

${ }^{24}$ F. Stern, Phys. Rev. Lett. 18, 546 (1967).

${ }^{25} \mathrm{H}$. Haug and S. W. Koch, Quantum Theory of the Optical and Electronic Properties of Semiconductors (World Scientific, Singapore, 1994).

${ }^{26}$ L. Wendler, F. Bechstedt, and M. Fiedler, Phys. Status Solidi B 159, 143 (1990).

${ }^{27}$ A.B. Henriques, Phys. Rev. B 44, 3340 (1991).

${ }^{28}$ H.N. Spector, J. Lee, and P. Melman, Superlattices Microstruct. 1, 149 (1985).

${ }^{29}$ J. Lee, H.N. Spector, and P. Melman, J. Appl. Phys. 58, 1893 (1985).

${ }^{30}$ W.S. Edelstein and H.N. Spector, Surf. Sci. 224, 581 (1989).

${ }^{31}$ J.A. Reyes and M. del Castillo-Mussot, Phys. Rev. B 56, 14893 (1997).

${ }^{32}$ M.E. Portnoi and I. Galbraith, Solid State Commun. 103, 325 (1997).

${ }^{33}$ N. Levinson, K. Dan. Vidensk. Selsk. Mat. Fys. Medd. 25, 3 (1949).

${ }^{34}$ R. G. Newton, Scattering Theory of Waves and Particles (McGraw-Hill, New York, 1966).

${ }^{35}$ For $m=1$, when a half-bound state occurs, $\nu_{m}$ denotes the number of bound states plus 1 . The wave function of the half-bound state tends to zero at infinity, although it does not decay fast enough at infinity to be square integrable. More on this subject can be found in Refs. 36 and 37.

${ }^{36}$ D. Bollé, F. Gesztesy, C. Danneels, and S.F.J. Wilk, Phys. Rev. Lett. 56, 900 (1986).

${ }^{37}$ Shi-Hai Dong, Xi-Wen Hou, and Zhong-Qi Ma, Phys. Rev. A 58, 2790 (1998).

${ }^{38}$ Q.-G. Lin, Phys. Rev. A 56, 1938 (1997); 57, 3478 (1998).

${ }^{39}$ V. Bargmann, Proc. Natl. Acad. Sci. USA 38, 961 (1952); see also M. Reed and B. Simon, Analysis of Operators (Academic, New York, 1978).

${ }^{40}$ W.G. Gibson, Phys. Rev. A 36, 564 (1987).

${ }^{41}$ A.I. Larkin, Zh. Éksp. Teor. Fiz. 38, 1896 (1960) [Sov. Phys. JETP 11, 1363 (1960)].

${ }^{42}$ F.J. Rogers, H.C. Graboske, Jr., and H.E. DeWitt, Phys. Lett. 34A, 127 (1971); F.J. Rogers, Phys. Rev. A 4, 1145 (1971).

${ }^{43}$ Surprisingly, the situation changes drastically in the zero-density limit $\left(q_{s} a^{*} \rightarrow 0\right.$, while $\left.k_{B} T / \mathrm{Ry}^{*} \sim 1\right)$. It can be shown that, in this limit $Z_{e h}+Z_{e e}=N_{b}+f\left(k_{B} T / \mathrm{Ry}^{*}, q_{s} a^{*}\right)$, where $\left|f\left(k_{B} T / \mathrm{Ry}^{*}, q_{s} a^{*}\right)\right| \ll N_{b}, N_{b}$ being the number of bound states. Then, using Eqs. (20) and (15), we obtain $\alpha \rightarrow 2 / 3$, when $q_{s} a^{*}$ $\rightarrow 0$, whereas a simple, single-bound-state law of the mass action gives $\alpha \rightarrow 1$ in the same limit.

${ }^{44}$ M.E. Portnoi and I. Galbraith, J. Cryst. Growth 184/185, 676 (1998).

${ }^{45}$ R.L. Siddon and M. Schick, Phys. Rev. A 9, 907 (1973); J. G. Dash, Films on Solid Surfaces (Academic, New York, 1975). 